\title{
Régime et comportement alimentaire du gardon (Rutilus rutilus (L.)) et de la perche (Perca fluviatilis L.) de la retenue de Pareloup. - I. Les alevins.
}

Diet and feeding behavior of roach (Rutilus rutilus [L.]) and perch (Perca fluviatilis L.) in Pareloup reservoir. - I. fry

\author{
C. Dubois( ${ }^{(*)}$, C. Richeux( $\left(^{(*)}\right.$, J.N. Tourenq( ${ }^{(*)}$ et C. Lejolivet $\left(^{(*)}\right.$ \\ (") Laboratoire d'Hydrobiologie, URA 695 C.N.A.S., Université Paul Sabatier, 118 route de \\ Narbonne. F-31062 Toulouse Cedex, France. \\ (*) Laboratoire d'lchthyologie Appliquée de Toulouse (ENSAT), 145 Avenue de Muret, \\ F-31076 Toulouse Cedex, France.
}

Résumé. - Le régime alimentaire des alevins de perches et de gardons du lac de Pareloup a été mis en évidence par l'analyse de leurs contenus intestinaux de juin à octobre des années 1985 et 1986. Quatre phases d'alimentation, en fonction de leur âge, peuvent être distinguèes chez ces alevins qui se nourrissent essentiellement de zooplancton et de macroinvertébrés.

Une série d'expériences sur leurs préférences alimentaires vis-à-vis du zooplancton fût réalisée durant l'été 1988 en conditions semi-naturelles. II apparait que les jeunes gardons sont très sélectifs vis-à-vis des Cladocères, contrairement aux jeunes perches. En revanche, ces dernières fournissent un effort de sélection important vis-à-vis des proies de grande taille, du moins jusqu'à la fin du mois d'août. A partir de septembre, ces tendances semblent s'inverser : les gardons n'ont plus de préférence exclusive pour les Cladoceres (les Copépodes sont aussi sélectionnés), et les proies de grande taille sont préférentiellement consommées. Inversement chez les perches, seuls les Cladocères sont sélectionnés positivement, et on assiste à une diminution de la préférence pour les proies de grande taille.

L'interdépendance entre les deux critères: taille des proies et position taxonomique est discutée, ainsi que l'effet de la compétition pour la nourriture entre les alevins de perches et de gardons.

Mots-clés. - Rutilus rutilus (L.), Perca fluviatilis L., régime alimentaire, contenus intestinaux, préférence alimentaire, zooplancton, compétition interspécifique. 
Abstract. - The diet of perch and roach fry in Pareloup Lake was determined by analysis of their intestinal content from June to October 1985 and 1986. The results indicate that these young fish feed essentially on zooplankton and macroinvertebrates. Four diet phases may be distinguished, depending on the age of the fry.

A series of experiments on the diet preferences of fry with respect to zooplankton was carried out under semi-natural conditions in summer 1988.

It appears that young roach are highly selective with regard to Cladocerans, unlike young perch. Perch, on the other hand, are extremely selective with regard to large-size prey, at least until the end of August. From September onwards, these trends seem to be inversed: roach no longer show an exclusive preference for Cladocerans (but also select Copepods), and prefer to consume large-size prey. Conversely, for perch, only Cladocerans are positively selected, and there is a decreased preference for large-size prey. This paper discusses the interdependence between these two criteria, size of prey and taxonomical position, as well as the effect of competition for nourishment between roach and perch fry.

Key-words. - Rutilus rutilus (L), Perca fluviatilis L. diet, intestinal contents, food preference, zooplankton, interspecific competition.

\section{INTRODUCTION}

L'estimation de l'impact d'un peuplement piscicole sur la structure des communautés de niveaux trophiques inférieurs passe par l'évaluation de la pression de prédation exercée sur ces derniers.

Pour cela, des données sur le comportement et le régime alimentaire des poissons sont nécessaires.

Dans cette étude, nous nous sommes intéressés aux alevins des deux espèces les mieux représentées dans le lac de Pareloup: le gardon et la perche.

Nous présentons ici la synthèse de deux travaux menés indépendamment: l'un portant sur le régime alimentaire (la nourriture effectivement ingérée), l'autre sur la préférence alimentaire (le choix éventuel des proies).
En effet, il est généralement admis que la plupart des alevins sont des prédateurs visuels qui chassent activement leurs proies (souvent planctoniques) après les avoir repérées visuellement (Crowder, 1985; Lazzaro, 1987).

Hrbacek (1962) et Brooks et Dodson (1965) ont été les premiers à mettre en évidence l'influence de tels prédateurs. Leurs résultats indiquent qu'ils modifient la structure de la communauté zooplanctonique de sorte que celle-ci se trouve composée d'espèces et d'individus de plus petite taille.

La prédation de ces poissons est donc sélective et se manifeste à travers la taille des proies (Werner et Hall, 1974; Confer et Blades, 1975; O'Brien et al., 1976): les proies les plus grosses sont préférées car elles sont plus facilement repérables et plus énergétiques. 
Cependant, la taille des organismes zooplanctoniques n'est probablement pas le seul critère de sélection : il est nécessaire de prendre en compte d'autres facteurs comme la pigmentation (Zaret et Kerfoot, 1975; Confer et al., 1978, 1980; Kerfoot, 1980), le type de locomotion (Zaret, 1980) et la capacité d'échappement (Confer et Blades, 1975; Drenner et al., 1978, 1980) des différentes proies.

L'apprentissage, l'expérience et la capacité d'acclimatation de la part du poisson interviennent également dans la sélection des proies (Furnass, 1979; Gardner, 1985).

La prédation de ces poissons visuels résulte donc d'un produit complexe de nombreux paramètres (O'Brien, 1979; Lazzaro, 1987).

Dans notre cas, nous nous sommes limités pour l'étude de la sélectivité aux proies zooplanctoniques, appréhendées en fonction de leur taille d'une part et de leur position taxonomique d'autre part; ceci dans le but de compléter les informations obtenues sur le régime alimentaire.

\section{MATÉRIEL ET MÉTHODES}

\section{Régime alimentaire}

Les alevins ont été capturés chaque mois de juin à octobre durant deux années consécutives $(1985,1986)$ par pêches électriques à proximité des frayères. Pour l'analyse des contenus intestinaux, les individus péchés (en- tre 10 et 20 à chaque campagne) sont conservés dans du formol à $10 \%$ après avoir été mesurés $( \pm 1 \mathrm{~mm})$ et pesés $( \pm 0,5 \mathrm{mg})$.

Les résultats obtenus chez les alevins de perches sont présentés sous forme d'un indice: I'IRI (Indice d'Importance Relative) proposé par Pinkhas et al. (1971), assez bien représentatif du régime car il combine les fréquences d'occurrence $(O C)$, numériques $(\mathrm{N})$ et pondérales $(\mathrm{W})$ de chaque type de proie:

IRli $(\%)=(\mathrm{Ni}+\mathrm{Wi}) \cdot \mathrm{OCi} / \sum_{i=1}^{n}(\mathrm{Ni}+\mathrm{Wi}) \cdot \mathrm{OCi}$

avec :

$\mathrm{Ni}=$ Nombre total d'individus de la catégorie de proie $\mathrm{i}$.

$W_{i}=$ Poids sec moyen (en $\mu \mathrm{g}$ ) d'un individu de la catégorie i.

$\mathrm{OCi}=$ Proportion (en \%) d'estomacs renfermant une proie i sur l'ensemble des estomacs renfermant de la nourriture.

$\mathbf{n}=$ somme des $\mathrm{i}$ catégories de proies.

Cet indice nécessite toutefois une détermination de tous les items ainsi que leur quantification pondérale individuelle.

Ceci s'est avéré particulièrement délicat chez les alevins de gardons en raison de la petite taille des proies et de leur état de dégradation suite à l'action des dents pharyngiennes, si bien qu'il fût préféré la méthode des points proposée par Swynnerton et Worthington (1940) adaptée au gardon par Linfield (1980). Elle consiste à attribuer trois notes $(\mathrm{F}, \mathrm{C}, \mathrm{Pi})$ variant 
de 0 à 10 dont les valeurs sont définies dans le tableau 1 :

$$
\% \text { Points } \mathrm{i}=\sum_{j-1}^{n}\left(\mathrm{Fj}_{\mathrm{j}} \cdot \mathrm{Cj} \cdot \mathrm{Pi}\right) / \sum_{i}\left(\mathrm{Fj} \cdot \mathrm{Cj}_{\mathrm{j}}\right)
$$

où :

Fj caractérise l'état de remplissage du tube digestif $j$.

Cj mesure l'importance relative de la nourriture assimilable dans le contenu du tube digestif $\mathrm{j}$.

Pi mesure la part de la proies i dans la nourriture assimilable contenue dans le tube digestif $\mathrm{j}$.

$\mathrm{n}$ est la somme des $\mathrm{j}$ tubes digestifs analysés.

\section{Préférence alimentaire}

L'évaluation du degré de sélectivité des poissons vis-à-vis des proies disponibles a nécessité une approche expérimentale différente de celle utilisée pour l'étude du régime alimentaire.

Réalisée durant l'été et l'automne 1988, une série d'expériences a été conçue dans le but de suivre au sein d'un mésocosme clos l'évolution de la densité de différents organismes zooplanctoniques sous l'action d'alevins prédateurs (perches et gardons).

Pour cela, des enceintes cylindriques en plastique rigide (hauteur = $1,85 \mathrm{~m}$, diamètre $=1,10 \mathrm{~m}$, volume d'eau $=1400 \mathrm{I}$ ), furent disposées dans le lac à une dizaine de mètres de la berge. Chacune d'entre elles est caractérisée par son contenu initial en alevin (tableau 2) et en zooplancton (tableau 3) (les enceintes sont enrichies en zooplancton dans des proportions taxonomiques indentiques au milieu naturel). Puis à temps régulier, sur un cycle de $24 \mathrm{~h}$, un échantillonnage est effectué (2 prélèvements de $5 \mathrm{I})$.

L'évaluation de la préférence alimentaire a été quantifiée à l'aide du Normalized Forage Ratio (NFR), indice proposé par Paloheimo (1979):

$$
\mathrm{NFRi}=(\mathrm{Ri} / \mathrm{Pi}) / \sum_{i=1}^{n}(\mathrm{Ri} / \mathrm{Pi})
$$

avec :

$\mathrm{Pi}=$ proportion de la proie $\mathrm{i}$ dans l'enceinte (environnement).

Tableau I. - Définition et valeurs des coefficients F, C et Pi (méthode des points).

Table I. - Definition and values of coefficients F, C and Pi (method of points).

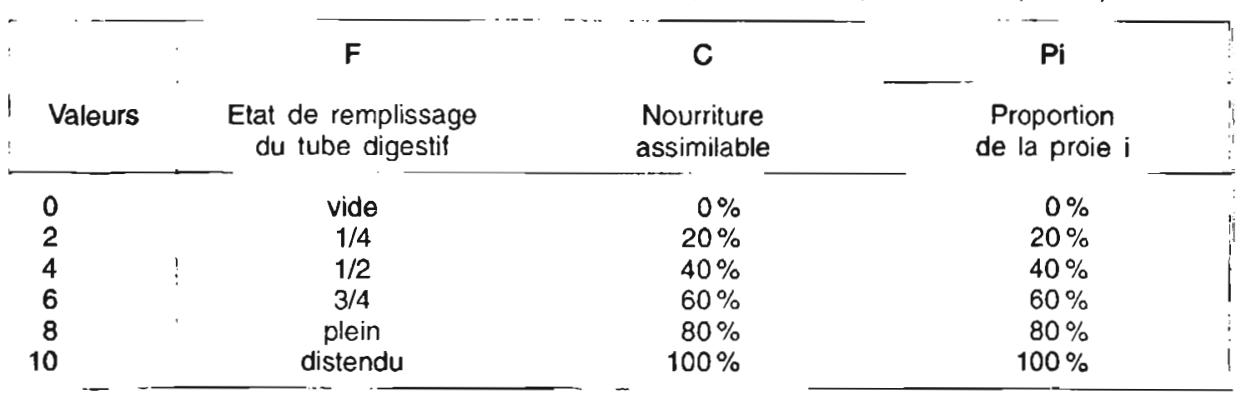


Tableau II. - Contenu des enceintes à chaque date d'expérience ( $\mathrm{N}=$ nombre d'alevins ; $\mathrm{Lt}=$ longueur totale moyenne en $\mathrm{mm}$ ). Une enceinte-témoin dépourvue d'alevins est suivie à chaque expérience.

Table II. - Contents of the enclosures on each experiment date ( $N=$ number of fry; Lt = average total length in $\mathrm{mm}$ ). For each experiment, one empty enclosure is monitored for the purposes of comparison.

\begin{tabular}{|c|c|c|c|c|}
\hline Date & 17.07 .88 & 30.08 .88 & 01.10 .88 & 25.10 .88 \\
\hline Gardons N & - & 22 & 35 & 25 \\
\hline $\mathrm{Lt}$ & - & 41 & 48 & 51 \\
\hline Perches $\mathbf{N}$ & 25 & 22 & 22 & - \\
\hline $\mathrm{Lt}$ & 33 & 42 & 55 & - \\
\hline
\end{tabular}

Tableau III. - Composition moyenne des enceintes en zooplancton (moyennes obtenues sur l'ensemble des enceintes de chaque expérience). - Densité (ind/l) et proportion (\%) de chaque classe de taille $(A, B, C, D)$.

Table III. - Average zooplankton composition in enclosures (averages obtained from all enclosures in a single experiment). - Density (ind./) and proportion (\%) of each size class (A, B, C, D).

\begin{tabular}{|c|c|c|c|c|c|}
\hline & $\begin{array}{l}\text { densité } \\
\text { (ind/l) }\end{array}$ & $\begin{array}{c}A \\
(\%)\end{array}$ & $\begin{array}{c}B \\
(\%)\end{array}$ & $\begin{array}{c}C \\
(\%)\end{array}$ & $\begin{array}{c}D \\
(\%)\end{array}$ \\
\hline \multicolumn{6}{|l|}{ Cladocères } \\
\hline D. longispina & 21,9 & 9,0 & 72,2 & 18,8 & 0,0 \\
\hline C. quadrangula & 58,4 & 29,2 & 70.1 & 0,7 & 0,0 \\
\hline Bosminidae & 6,8 & 93,1 & 6,9 & 0,0 & 0,0 \\
\hline Sididae & 18,0 & 12,2 & 87,1 & 0,7 & 0,0 \\
\hline Autres & 4,1 & 58,8 & 40,0 & 1,2 & 0,0 \\
\hline \multicolumn{6}{|l|}{ Copépodes } \\
\hline Cyclops & 7,4 & 0,0 & 83,7 & 15,2 & 1,1 \\
\hline Diaptomus & 25,4 & 0,0 & 67,2 & 31,1 & 1,7 \\
\hline Nauplius & 13,6 & 100,0 & 0,0 & 0,0 & 0,0 \\
\hline Rotifères & 65,7 & 100,0 & 0,0 & 0,0 & 0,0 \\
\hline
\end{tabular}

$\mathrm{R} i=$ proportion de la proie $\mathrm{i}$ dans le régime du poisson. Ri est obtenu par différence entre $\mathrm{Pi}$ initial et $\mathrm{Pi}$ final (intervalle de temps $\approx=24 \mathrm{~h}$ ), compte-tenu de la mortalité naturelle (déduite à partir d'une enceinte témoin, sans alevins).

$\mathrm{n}=$ ensemble des proies, considérées comme tout organisme consommé.
Cet indice exprime la proportion du rapport $\mathrm{Ri} / \mathrm{Pi}$ (Forage Ratio de Edmonson et Winberg, 1971) de chaque type de proie i consommé : lorsque ce rapport est supérieur à $1(\mathrm{Ri}>\mathrm{Pi})$, la sélection de la proie i est positive.

Le NFR est donc un indice de "préférence relative", qui "normalisé" (indépendant de l'abondance re- 
lative Pi), a l'avantage de traduire le degré de préférence et de permettre des comparaisons entre milieux différents.

Nous avons choisi deux axes, traités indépendamment, dans la caractérisation des types de proies (Tableau 3) :

- le groupe zoologique,

- la taille : les proies zooplanctoniques furent rangées en classe de taille comme suit:

Classe A : $<400 \mu \mathrm{m}$

B : $400-800 \mu \mathrm{m}$

C : $800-1200 \mu \mathrm{m}$

D : > $1200 \mu \mathrm{m}$.

\section{RÉSULTATS}

\section{Régime alimentaire}

\section{Gardons}

L'analyse descriptive des contenus intestinaux des jeunes gardons de l'année a permis de mettre en évidence quatre périodes d'alimentation (tableau 4).

De 10 à $15 \mathrm{~mm}$ (mi-juin), le régime est très peu diversifié et est composé essentiellement de petites proies (Rotifères, Protistes) (tableau 4).

De 15 à $30 \mathrm{~mm}$ (juillet), l'alimentation est plus riche et à base de larves d'insectes (Diptères Chironomidae essentiellement). Le zooplancton fait figure de nourriture secondaire. On note également une proportion importante d'Annelides.
De 30 à $40 \mathrm{~mm}$ (août), le régime est toujours composé principalement de larves d'insectes: on observe une diminution de la contribution des Chironomidae au profit des Hétéroptères. Une quantité non négligeable de phytoplancton a été observée ainsi qu'une forte ingestion de matière en suspension et autres débris organiques (non quantifié).

Au delà de $40 \mathrm{~mm}$ (à l'automne), les alevins reviennent à une alimentation moins diversifiée à base de petites proies zooplanctoniques (Chydoridae ou Bosminidae et secondairement Daphniidae).

\section{Perches}

L'analyse des bols alimentaires des alevins de perches a permis de distinguer également quatre phases d'alimentation (tableau 5).

Les perches inférieures à $20 \mathrm{~mm}$ (juin) sont essentiellement zooplanctophages, les Cladocères représentant l'item principal $(72 \%$ du bol dont $49 \%$ de Daphniidae et $20 \%$ de Sididae). Les proies secondaires sont les Cyclopidae $(10,2 \%)$, des larves de Cyprinidae $(8,6 \%)$ et des Chironomidae $(7,7 \%)$.

Entre 25 et $35 \mathrm{~mm}$ (juillet), le zooplancton domine encore l'alimentation ( $54 \%$ en $1985,50,1 \%$ en 1986 ) mais la part des macroinvertébrés est également importante $(40,6 \%$ en 1985 et $49,8 \%$ en 1986). Parmi les items zooplanctoniques, ce sont les Cyclopidae (Copépode) qui constituent toujours la proie principale $(\approx 30 \%)$ avec les Chydoridae et/ou les Daphniidae (Clado- 
Tableau IV. - Rutilus rutilus. - Valeur de l'indice des points obtenue pour chaque type de proies.

Table IV. - Rutilus rutilus. - value of the point index for each type of prey.

\begin{tabular}{|c|c|c|c|c|c|c|c|c|c|c|}
\hline \multirow[b]{2}{*}{ PROIES } & \multicolumn{6}{|c|}{1985} & \multicolumn{4}{|c|}{1986} \\
\hline & $19 / 06$ & $12 / 07$ & $25 / 07$ & $15 / 08$ & $06 / 09$ & $15 / 10$ & $14 / 06$ & $08 / 07$ & $29 / 08$ & $16 / 10$ \\
\hline \multicolumn{11}{|l|}{ ZOOPLANCTON } \\
\hline \multicolumn{11}{|l|}{ Cladocères } \\
\hline Bosminidae & 0 & 0 & 2,1 & 2 & 0,7 & 0,3 & 8,2 & 1 & 0,5 & 40,4 \\
\hline Chydoridae & 0,9 & 9,9 & 5,1 & 16,4 & 42,6 & 63,6 & 2,3 & 4,7 & 13,9 & 15,5 \\
\hline Sididae & 0 & 0 & 0 & 0 & 0 & 0 & 0 & 0 & 3,3 & 0,2 \\
\hline Daphniidae & 16,9 & 0 & 8,9 & 10,9 & 1,6 & 0,3 & 0 & 12,6 & 5,4 & 12,4 \\
\hline \multicolumn{11}{|l|}{ Copépodes } \\
\hline Cyclopidae & 1 & 6 & 4,2 & 7,8 & 30,8 & 1,8 & 0 & 3,6 & 1,7 & 0,9 \\
\hline Calanidae & 0 & 0,3 & 0 & 0 & 0 & 4,6 & 0 & 0 & 0 & 0 \\
\hline Rotifères & 30,6 & 0,1 & 0 & 0 & 0 & - & 33,5 & 9,8 & - & 0 \\
\hline \multicolumn{11}{|c|}{ MACROINVERTÉBRÉS } \\
\hline Chironomidae & 9,8 & 12,9 & 14 & 10,8 & 5,4 & 21,5 & 5,7 & 50,9 & 37 & 6 \\
\hline Héteroptères & 0 & 4,1 & 2,7 & 15,1 & 0 & 0 & 0 & 0 & 9,5 & 0 \\
\hline Plécoptères & 0 & 0 & 1,5 & 0 & 0 & 0 & 0 & 0 & 1,2 & 0 \\
\hline Insectes N.D. & 0 & 0 & 16,1 & 16,2 & 13,3 & 1,3 & 0 & 1,4 & 9,7 & 5 \\
\hline Acariens & 0 & 5,2 & 3,4 & 0 & 0,2 & 0 & 0 & 0 & 1,7 & 0 \\
\hline Annélides & 0 & 47,9 & 33,3 & 2,4 & 0 & 0 & 0 & 9,6 & 3,4 & 0 \\
\hline Indéterminés & 0 & 0 & 0 & 0 & 0 & 0,3 & 0 & 1,1 & 0,5 & 18,7 \\
\hline \multicolumn{11}{|l|}{ ALGUES } \\
\hline coloniales & 0 & 6,2 & 2,9 & 4,4 & 0,2 & 0,2 & 4,1 & 1,3 & 0,8 & 0 \\
\hline cellulaires & 8,8 & 5,7 & 4,4 & 7,5 & 5 & 6,1 & 2,3 & 2,7 & 11,3 & 0,7 \\
\hline filamenteuses & 0 & 0,1 & 0,2 & 1,2 & 0 & - & 0,6 & 0,4 & 0.4 & 0,3 \\
\hline \multicolumn{11}{|l|}{ AUTRES } \\
\hline Ciliés & 0 & 1,2 & 0,4 & 0,4 & - & 0,2 & 0 & - & 0 & 0 \\
\hline Thécamébiens & 31,8 & 0 & 0 & 0 & 0 & 0 & 13,9 & 1 & 0 & 0 \\
\hline Dinoflagellès & 0 & 0 & 0 & 0 & 0 & 0 & 29,3 & 0 & 0 & 0 \\
\hline
\end{tabular}

cères). Les Chironomidae représentent le principal macroinvertébré ingéré.

Les perches de 35 à $55 \mathrm{~mm}$ ingèrent essentiellement soit du zooplancton (Sididae et Cyclopidae) soit des macroinvertébrés (Chironomidae et Baetidae) selon le lieu de pêche et vraisemblablement de la disponibilité du milieu en proies.
A l'automne lorsque la taille des alevins dépasse $55 \mathrm{~mm}$, le zooplancton redevient source de nourriture principale. Les Copépodes dominent dans tous les cas (Cyclopidae et/ou Diaptomidae); les macroinvertébrés (septembre 1985) ou les Cladocères (octobre 1986) représentent la seconde source de nourriture. 
Tableau V. - Perca fluviatilis: Valeur de I'IRI (\%) obtenue pour chaque type de proies $(P=$ présence). Aux dates 29.08 .86 et 14.10 .86 , deux frayères diffèrentes furent prospectées.

Table V. - Perca fluviatilis: Value of the IRI $(\%)$ for each type of prey ( $P=$ presence). On 29 August 86 and 14 October 86 , two separate spawning grounds were prospected.

\begin{tabular}{|c|c|c|c|c|c|c|c|c|c|}
\hline \multirow[b]{2}{*}{ PROIES } & \multicolumn{4}{|c|}{1985} & \multicolumn{4}{|c|}{1986} & $-\square$ \\
\hline & $20 / 06$ & Juillet & $15 / 08$ & $06 / 09$ & $08 / 07$ & $29 / 08^{*}$ & $29 / 08^{* *}$ & $14 / 10^{*}$ & $14 / 10^{* *}$ \\
\hline \multicolumn{10}{|l|}{ ZOOPLANCTON } \\
\hline \multicolumn{10}{|l|}{ Cladocères } \\
\hline Bosminidae & 0,8 & 0,3 & 0 & 0 & 0,1 & 0 & 0 & 3,8 & 7,4 \\
\hline Chydoridae & 1,5 & 23,6 & 0 & 1,8 & 7,2 & 1 & 2,4 & 0,8 & 5,7 \\
\hline Sididae & 20,3 & 0 & 0 & 0 & 1 & 40,2 & 43,8 & 0,3 & 1,8 \\
\hline Daphniidae & 49,1 & 2,2 & 0 & 0 & 10,9 & $P$ & 3,1 & 2,5 & 13,7 \\
\hline \multicolumn{10}{|l|}{ Copépodes } \\
\hline Cyclopidae & 10,2 & 28,4 & 0 & 76,6 & 30,9 & 1 & 24,6 & 0,8 & 49,7 \\
\hline Diaptomidae & 0 & 0 & 0 & 0 & 0 & 0 & 0 & 83,4 & 5,9 \\
\hline \multicolumn{10}{|c|}{ MACROINVERTÉBRÉS } \\
\hline Chironomidae & 7,7 & 34,2 & 0 & 0 & 49,7 & 35,7 & 17 & 1,8 & $\mathbf{P}$ \\
\hline Baetidae & 0 & 0 & 0 & 15,5 & 0 & 16,4 & 6,1 & $\mathbf{P}$ & $\mathbf{P}$ \\
\hline Hétéroptères & 1,7 & 4,8 & 0 & 6,1 & 0,1 & 5,6 & 0,8 & 0 & $\mathbf{P}$ \\
\hline Trichoptères & 0 & 0 & 0 & 0 & 0 & 0 & 2,2 & 6,3 & 0 \\
\hline Acariens & 0 & 1,6 & 0 & 0 & $\mathbf{P}$ & 0 & 0 & 0 & 0 \\
\hline Argulides & 8,6 & 4,8 & 0 & 0 & 0 & 0 & 0 & 0 & 15,6 \\
\hline
\end{tabular}

\section{Préférence alimentaire}

\section{Alevins de gardons}

II ne semble pas y avoir de changements notables dans l'évolution des préférences relatives de fin aôut à fin octobre, du moins en fonction de la position systématique des proies (fig. 1): les Cladocères sont toujours préférentiellement sélectionnés aux dépens des Copépodes; les Rotifères étant systématiquement "ignorés". Parmi les Cladoceres, Daphnia longispina semble être l'espèce préférée (pour toute les expériences, on a $\mathrm{Ri}$ $>$ Pi) (tab. 6). En revanche, les Bosminidae sont dans chaque cas rejetés $(\mathrm{Ri}<\mathrm{Pi})$. Parmi les Copépodes, no-

tons que les larves nauplii sont totalement ignorées jusqu'au début du mois d'octobre, puis deviennent la proie préférée fin octobre.

En fonction de la taille des proies (fig. 2), ce sont les individus de taille moyenne (classe B) qui sont préférentiellement sélectionnés. Cependant, en fin de période d'étude (25.10.88), on assiste à un accroissement de la sélection (qui devient positive) des proies les plus grosses (classe $C+D$ ).

\section{Alevins de perches}

Au début de la période d'étude (17.07.88), la préférence relative semble revenir plutôt aux Copépodes (fig. 3). Par la suite, la tendance s'in- 

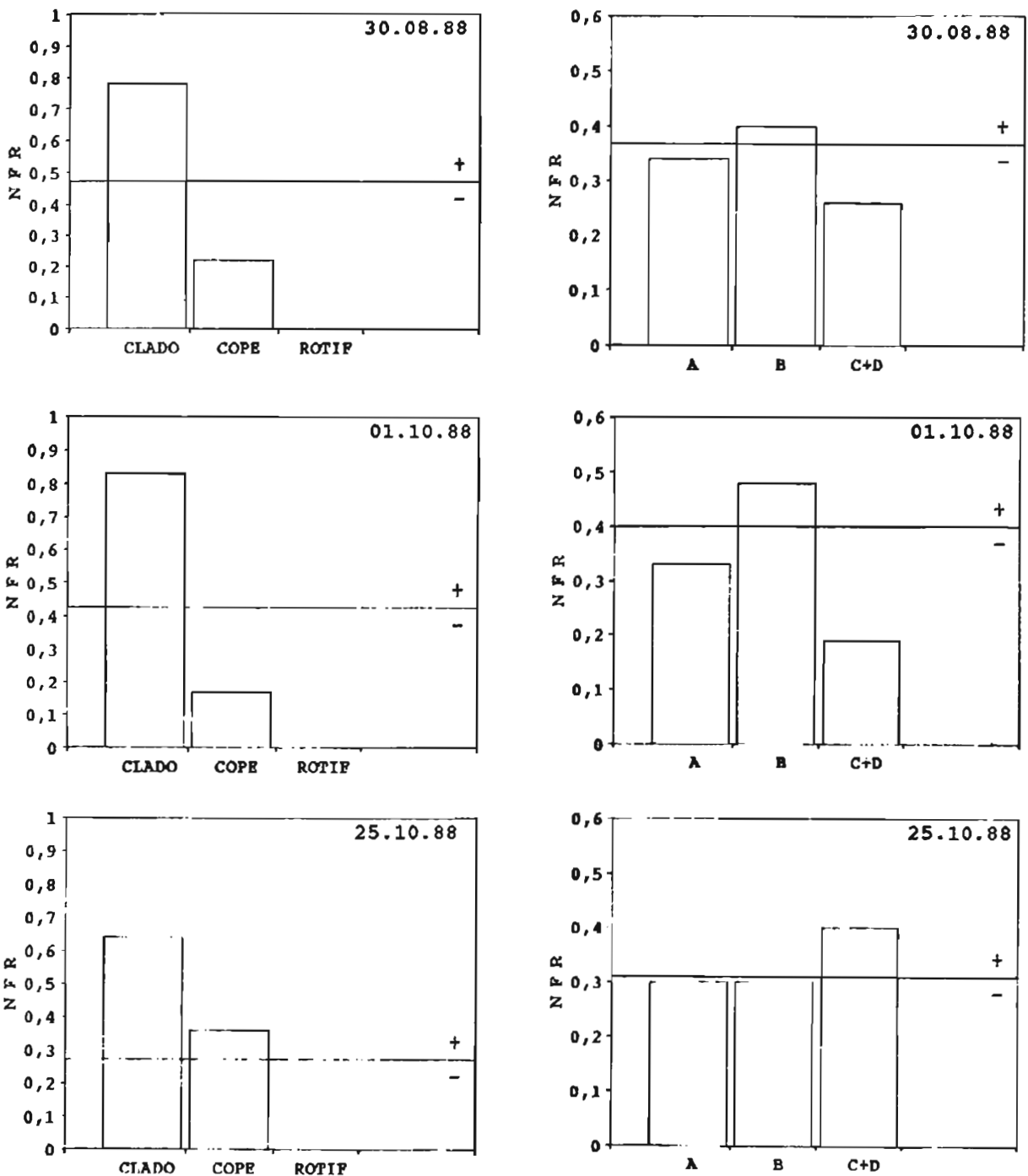

Fig. 1. - Rutilus rutilus : Evolution de la préférence (indice NFR) pour chaque type de proies (Cladocères, Copépodes, Rotiferes). La ligne horizontale correspond à la valeur du NFR pour laquelle $\mathrm{Ri}=\mathrm{Pi}$. Au dessus, la sélection est positive $(\mathrm{Ri}>$ $\mathrm{Pi})$; en dessous, elle est négative ( $\mathrm{Ri}<\mathrm{Pi}$ ).

Fig. 1. - Rutilus rutilus: evolution of the preference (NFR index) for each type of prey (Cla. docera, Copepodae, Rotifera). The horizontal line corresponds to the NFR value for which $\mathrm{Ri}=\mathrm{Pi}$. Above this line, the selection is positive $(\mathrm{Ri}>\mathrm{Pi})$; below this line, it is negative $(\mathrm{Ri}<\mathrm{Pi})$.

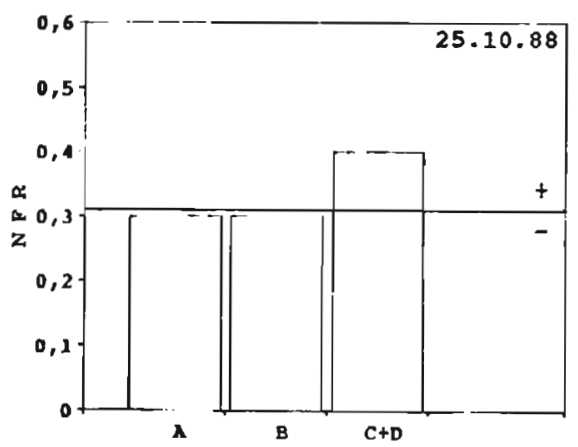

Fig. 2. - Rutilus rutilus: Evolution de la préférence (indice NFR) pour chaque classe de taille $(A, B, C+D)$. La ligne horizontale correspond à la valeur du NFR pour laquelle $\mathrm{Ri}=\mathrm{Pi}$. $\mathrm{Au}$ dessus, la sélection est positive $(\mathrm{Ri}>\mathrm{Pi})$; en dessous, elle est négative ( $\mathrm{Ri}<\mathrm{Pi}$ ).

Fig. 2. - Rutilus rutilus: evolution of the preference (NFR index) for each size-class ( $A, B$, $C+D)$. The horizontal line corresponds to the NFR value for which $\mathrm{Ri}=\mathrm{Pi}$. Above this line, the selection is positive ( $\mathrm{Ri}>\mathrm{Pi}$ ); below this line, it is negative $(\mathrm{Ri}<\mathrm{Pi})$. 
Tableau VI. - Rutilus rutilus: Valeur de l'indice NFR relatif à chaque taxon parmi les Cladocères et parmi les Copépodes. Les valeurs suivies d'un astérisque sont celles pour lesquelles $\mathrm{Ri}>\mathrm{Pi}$ (sélection positive).

Table VI. - Rutilus rutilus: Value of the NFR index for each taxon among Cladocerans and among Copepods. Values followed by an asterisk are those for which $\mathrm{Ri}>\mathrm{Pi}$ (positive selection).

\begin{tabular}{lccc} 
& & & \\
Taxons & 30.08 .88 & 01.10 .88 & 25.10 .88 \\
Cladocères & & & \\
D. longispina & $0,31^{*}$ & $0,26^{*}$ & $0,28^{*}$ \\
C. quadrangula & 0,21 & 0,16 & $0,19^{*}$ \\
Bosminidae & 0,13 & 0,18 & 0,12 \\
Sididae & 0,17 & 0,15 & $0,21^{*}$ \\
Autres & 0,19 & $0,25^{*}$ & $0,21^{*}$ \\
& & & \\
Copépodes & & $0,77^{*}$ & 0,00 \\
Cyclops & $0,30^{*}$ & $0,23^{*}$ & 0,15 \\
Diaptomus & $0,70^{*}$ & 0,00 & $0,85^{*}$ \\
Nauplius & 0,00 & & \\
\hline
\end{tabular}

Tableau VII. - Perca fluviatilis: Valeur de l'indice NFR relatif à chaque taxon parmi les Cladocères et parmi les Copépodes. Les valeurs suivies d'un astérisque sont celles pour lesquelles $\mathrm{Ri}>\mathrm{Pi}$ (sélection positive).

Table VII. - Perca fluviatilis: Value of the NFR index for each taxon among Cladocerans and among Copepods. Values followed by an asterisk are those for which RiPi (positive selection).

\begin{tabular}{llll|} 
Taxons & 17.07 .88 & 30.08 .88 & 01.10 .88 \\
Cladocères & & & $0,30^{*}$ \\
D. longispina & $0,23^{*}$ & $0,23^{*}$ & 0,20 \\
C. quadrangula & 0,15 & 0,21 & 0,23 \\
Bosminidae & 0,20 & 0,18 & $0,26^{*}$ \\
Sididae & 0,18 & $0,22^{*}$ & 0,01 \\
Autres & $0,24^{*}$ & 0,16 & \\
Copépodes & & & $0,42^{*}$ \\
Cyclops & 0,37 & $0,39^{*}$ & $0,47^{*}$ \\
Diaptomus & $0,49^{*}$ & $0,61^{*}$ & 0,11 \\
Nauplius & 0,14 & 0,00 & \\
\end{tabular}

verse et ce sont les Cladocères qui sont préférentiellement sélectionnés (30.08.88 et 01.10.88). Comme chez les gardons, les Rotifères sont ignorés. Parmi les Copépodes, le genre Diaptomus (le plus abondant) est toujours préféré au genre Cyclops (tab. 7). Les larves nauplii quant à elles sont globalement évitées. Concernant les Cladocères, $D$. longispina et les Sididae semblent être les taxons préférés, les Bosminidae étant dans tout les cas rejetés. 

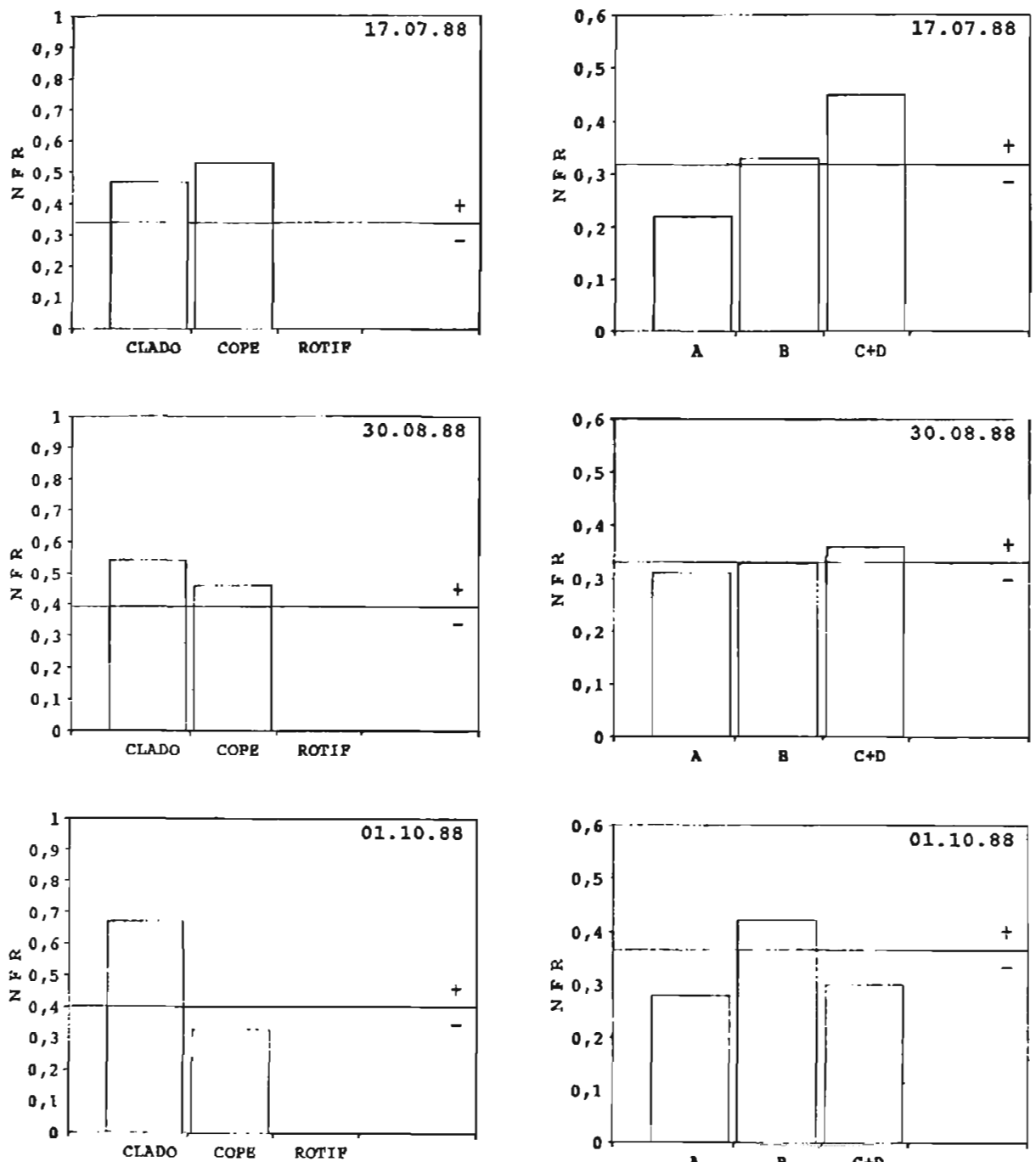

Fig. 3. - Perca fluviatilis: Evolution de la préférence (indice NFR) pour chaque type de proies (Cladoceres, Copépodes, Rotifères). La ligne horizontale correspond à la valeur du NFR pour laquelle $\mathrm{Ri}=\mathrm{Pi}$. Au dessus, la sélection est positive $(\mathrm{Ri}>\mathrm{Pi})$; en dessous, elle est négative $(\mathrm{Ri}<\mathrm{Pi})$.

Fig. 3. - Perca fluviatilis : evolution of preference (NFR index) for each type of prey (Cladocera, Copepodae, Rotifera). The horizontal line corresponds to the NFR value for which $\mathrm{Ri}=\mathrm{Pi}$. Above this line, the selection is positive $(\mathrm{Ri}>\mathrm{Pi})$; below this line, it is negative $(\mathrm{Ri}<\mathrm{Pi})$.

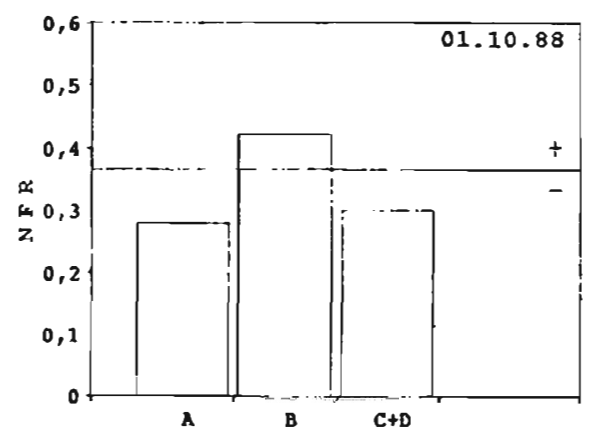

Fig. 4. - Perca fluviatilis : Evolution de la préférence (indice NFR) pour chaque classe de taille $(A, B, C+D)$. La ligne horizontale correspond à la valeur du NFR pour laquelle $\mathrm{Ri}=\mathrm{Pi}$. $\mathrm{Au}$ dessus, la sélection est positive $(\mathrm{Ri}>\mathrm{Pi})$ : en dessous, elle est négative $(\mathrm{Ri}<\mathrm{Pi})$.

Fig. 4. - Perca fluviatilis: evolution of the preference (NFR index) for each size-class (A, B, $C+D)$. The horizontal line corresponds to the NFR value for which $\mathrm{Ri}=\mathrm{Pi}$. Above this line, the selection is positive $(\mathrm{Ri}>\mathrm{Pi})$; below this line, it is negative ( $\mathrm{Ri}<\mathrm{Pi}$ ). 
En fonction de la taille (fig. 4), les proies les plus petites (classes $A$ et B) ne sont pas sélectionnées dans un premier temps. Cependant, en fin de la période d'étude $(01.10 .88)$, la valeur de l'indice NFR pour ces deux classes est légèrement plus forte, et ce sont les individus de taille moyenne qui sont préférés (classe $B$ ). L'augmentation de la sélection des Cladocères et la diminution de la sélection des proies de grande taille sont probablement à relier, la taille moyenne des Cladocères étant inférieure à celle des Copépodes (tab. 3).

\section{DISCUSSION}

\section{Régime alimentaire}

\section{Alevins de gardons}

La succession des phases d'alimentation observée chez les alevins de gardons est plus ou moins en accord avec les données bibliographiques:

La première source de nourriture est constituée essentiellement de Rotifères (Broughton et Jones, 1978; Hammer, 1985; Ponton, 1986), puis très vite les jeunes gardons ingèrent d'autres proies (Millikin, 1982). Les larves d'insectes de petite taille, mais surtout les crustacés planctoniques peuvent représenter une part non négligeable du régime chez les alevins de deux semaines et devenir les proies préférentielles des individus plus agés (Linfield, 1980; Niederholzer et Hofer, 1980; Boikova, 1986; Weatherley, 1987). A Pareloup, les larves d'insectes (Chironomidae puis Ephéméroptères et Hétéroptères) deviennent effectivement les proies principales dès la taille de $15 \mathrm{~mm}$.

Certains auteurs ont également constaté la faible importance voire l'absence du zooplancton chez les gardons de 6 à 10 semaines (Ponton, 1986; Weatherley, 1987). Dans notre cas cette absence n'est que temporaire et dès le mois d'août ( 8 semaines) le zooplancton réapparait dans le régime, puis devient majoritaire en septembre, comme dans le lac de Sainte Croix (Chappaz, 1986).

Un aspect particulier du régime des gardons, déjà signalé par la plupart des auteurs, est l'ingestion de végétaux (algues, macrophytes) et de détritus. A Pareloup, ils ne représentent jamais plus de $13 \%$ du matériel ingéré chez les gardons de l'année.

\section{Alevins de perches}

De nombreux auteurs se sont intéréssés au régime alimentaire des jeunes perches et considèrent tous qu'ils sont planctono-entomophages.

Le zooplancton constitue le régime des plus petits individus, mais dès l'âge de 3 mois environ, l'entomophagie devient prépondérante. A Pareloup, ces deux types de proies (zooplancton et insectes) se retrouvent lors des quatre phases.

Les plus petites perches (<20 mm) sont essentiellement planctonophages, et c'est à partir de $25 \mathrm{~mm}$ que les larves d'insectes apparaissent dans le tube digestif, conformément aux observations de Spanovskaya et 
Grygorash (1977) et Boikova (1986) dans des réservoirs de l'ex-Union Soviétique, Ney et al. (1975) dans des lacs américains.

Mais l'entomophagie peut apparaître plus tardivement: ce n'est qu'à des tailles de $61 \mathrm{~mm}$ (Paxton et Stevenson, 1978), 60 à $70 \mathrm{~mm}$ (Gerdeaux et Melaoui cités par Lesher Moutoue et al., 1975), ou $75 \mathrm{~mm}$ (Bregazzi et Kennedy, 1982) que les insectes constituent la source de nourriture principale. Dans notre cas, les alevins de cette taille $(60$ à $75 \mathrm{~mm}$ ) reviennent à un régime planctonophage (automne). Un phénomène identique est signalé dans le réservoir de Vestonicka (Kokes, 1984).

\section{Préférence alimentaire}

On observe, aussi bien chez le gardon que chez la perche, une selectivité dans la prise de nourriture. Ceci confirme l'hypothèse que ces alevins sont des prédateurs visuels.

Les alevins de gardons sont moins sélectifs vis-à-vis des grosses proies que les alevins de perches, jusqu'au début du mois d'octobre. Cependant, la taille des proies n'est sans doute pas le seul critère de sélection: en effet, les jeunes gardons, durant cette période, démontrent une préférence marquée pour les Cladocères. Or, la taille moyenne de ceux-ci est inférieure à celle des Copépodes (hormis les larves nauplii, de toute façon evitées). En revanche, il a été montré à plusieurs reprises que les Cladocères possédaient une capacité d'échap- pement aux prédateurs bien plus faible que les Copépodes (Confer et Blades, 1975; Drenner et al., 1978, 1980).

A la fin du mois d'octobre (25.10.88), la préférence des gardons revient clairement aux proies des classes de taille $C$ et $D$ (fig. 2), bien que l'essentiel des Copépodes sélectionnés soit des larves nauplii (tab. 6).

Par conséquent, l'évolution de la sélectivité par rapport à la taille des proies chez les jeunes gardons apparait indépendante du choix entre Cladocères et Copépodes.

Ceci ne semble pas être le cas chez les jeunes perches: la diminution de la préférence, en fin de période d'étude, pour les grosses proies est probablement à relier avec l'évitement progressif des Copépodes, au profit des Cladocères.

Ce changement à partir du mois d'octobre, dans la sélection des proies, correspond à la quatrième phase d'alimentation des deux espèces étudiées: les alevins de gardons et de perches reviennent en automne à un régime essentiellement composé de zooplancton en délaissant les macroinvertébrés; les jeunes gardons se nourrissant en grande part de Cladocères, les jeunes perches de Copépodes. Or nous avons vu que les alevins de ces deux espèces ont une nette préférence, à cette période, pour les Cladocères.

Persson $(1986,1987)$ a mis en évidence que les organismes zooplanctoniques préférés par les alevins de perches sont finalement toujours les 
Cladocères. Cependant les jeunes gardons semblent être des compétiteurs de ces perches, et les poussent vers un régime plus orienté vers les Copépodes et les invertébrés benthiques.

Cette répartition de la nourriture zooplanctonique (de façon globale: Cladocères pour les gardons, Copépodes pour les perches, que l'on observe bien dans l'étude du régime alimentaire) a également été notée par d'autres auteurs: Guma'a (1978) et Kokes (1984) montrent que les jeunes perches ingèrent préférentiellement les Copépodes. Persson (Op. cit.), Persson et Greenberg (1990) et Bergman (1990) considerent les alevins de gardons comme des planctophages très efficients, surtout au niveau des Cladocères.

La préférence alimentaire apparaît donc déterminée en partie par les caractéristiques du milieu (composition en proies, présence de compétiteurs) vis-à-vis desquelles le poisson s'acclimate (Furnass, 1979; Gardner, 1985). Si bien que les préférences constatées, d'après des expériences ponctuelles sur la sélectivité, suivent d'assez près le régime observé.

\section{RÉFÉRENCES BIBLIOGRAPHIQUES}

Bergman E., 1990. Effects of roach Rutilus rutilus on two percids, Perca fluviatilis and Gymnocephalus cernua: importance of species interactions for diets shifts. Oikos, 57 : 241-249.
Boikova O.S., 1986. Feeding of fish in Lake Glubokoe. Hydrobiologia, 141 : 95-111.

Bregazzi P.R. et Kennedy C.R., 1982. The responses of perch (Perca fluviatilis $L$.) population to eutrophication and associated changes in fish fauna in a small lake. J. Fish. Biol., $20:$ 21-31.

Brooks J.L. et Dodson S.I., 1965. Predation, body size and the composition of plankton. Science, $150:$ 28-35.

Broughton N.M. et Jones M.V., 1978. An investigation into the growth of 0-group roach (Rutilus rutilus (L.)) with special reference to temperature. J. Fish. Biol., 12 : 345-357.

Chappaz R., 1986. Etude piscicole de le retenue de Sainte Croix-Fontaine l'Evêque. Thèse (Doc. d'Univ.), Centre St Charles, $183 \mathrm{p}$.

Confer J.L. et Blades P.I., 1975. Omnivorous zooplankton and planktivorous fish. Limnol. Oceanogr., 20 : 571-579.

Confer J.L., Howick G.L., Corzette M.H., Kramer S.L., Fitzgibbon S. et Landesberg R., 1978. Visual predation by planktivores. Oikos, 31:27-37.

Confer J.L., Applegate G. et Evanik C.A., 1980. Selective predation by zooplankton and the response of cladoceran eyes to light. In: W.C. Kerfoot (ed.), Evolution and Ecology of Zooplankton Communities. The Univ. Press of New England, Hanover, New Hampshire, USA : 604-608.

Crowder L.B., 1985. Optimal foraging and feeding mode shifts in fishes. Envir. Biol. Fishes, 12 (1) : 57-62.

Drenner R.W., Strickler J.R. et O'Brien W.J., 1978. Capture probability. The role of zooplankter escape in the selective feeding of planktivorous fish. J. Fish. Res. Board Can., 35 (10) : 13701373.

Drenner R.W. et Mc Comas S.R., 1980. The role of zooplankter escape ability in the selective feeding and impact of planktivorous fish. In: W.C. Kerfoot (ed.), Evolution and Ecology of Zoo- 
plankton Communities. The Univ. Press New England, Hanover, New Hampshire, USA : 587-593.

Edmondson W.T. et Winberg G.G. (eds.), 1971. A manual on methods for the assessment of secondary productivity in fresh waters. IBP Handbook no. 17, Backwell, Oxford, $368 \mathrm{pp}$.

Furnass T.I., 1979. Laboratory experiments on prey selection by perch fry (Perca fluviatilis). Freshwat. Biol., 9: 33-43.

Gardner M.B., 1981. Mechanism of size selectivity by planktivorous fish : A test of hypotheses. Ecology, 62 : 571-578.

Guma'a S.A., 1978. The food and feeding habits of young perch, Perca fluviatilis, in Windermere. Freshwat. Biol., 8: 177-187.

Hammer C., 1985. Feeding behaviour of roach (Rutilus rutilus) larvae and the fry of perch (Perca fluviatilis) in lake Lankau. Arch. Hydrobiol., 103 : 61-74.

Hrbacek J., 1962. Species composition and the amount of zooplankton in relation to the fish stock. Rozpr. Cesk. Akad. Ved., 72 : 1-117.

Kerfoot W.C., 1980. Commentary: Transparency, body size and prey conspiciousness. In: W.C. Kerfoot (ed.), Evolution and Ecology of Zooplankton Communities. The Univ. Press of New England, Hanover, New Hampshire, USA : 609-617.

Kokes J., 1984. The food of the fry of perch (Perca fluviatilis) in the Musov reservoirs. Fol. Zool., 33 : 349-362.

Lazzaro X., 1987. A review of planktivorous fishes: their evolution, feeding behaviours, selectivities, and impacts. Hydrobiologia, 146 : 97-167.

Lescher-Moutoué F., Garnier J. et Pourriot R., 1985. Interactions entre les peuplements planctonique et piscicole du lac de Créteil : impact d'une reproduction exceptionnelle de percidés. Bull. Ecol., 16 (1) : 9-17.

Linfield R.S., 1980. Ecological changes in a lake fishery and their effects on a stunted roach (Rutilus rutilus) population. J. Fish. Biol., $16:$ 123-144.

Millikin M.R., 1982. Qualitative and quantitative nutrient requirement of fishes: a review. Fish. Bull., 80:655-686.

Ney J.J., Lloyd L. et Smith J.R., 1975. First-year growth of the yellow perch, Perca flavescens, in the Red Lakes, Minnesota. Trans. Am. Fish. Soc., 104 (4) : 718-725.

Niederholzer R. et Hofer R., 1980. The feeding of roach (Rutilus rutilus (L.)) and rudd (Scardinius erythrophthalmus (L.)). I. Studies on natural populations. Ekol. Pol., 28 (1) : 45-59.

O'Brien W.J., Slades N.A. etr Vinyard G.L., 1976. Apparent size as the determinant of prey selection by bluegill sunfish (Lepomis macrochirus). Ecology, 57 : 1304-1310.

O'Brien W.J., 1979. The predatory-prey interaction of planktivorous fish and zooplakton. American scientist, 67 : 572-581.

Paloheimo J.E., 1979. Indices of food type preference by a predator. J. Fish. Res. Board Can., $36:$ 470-473.

Paxton K.O. et Stevenson F., 1978. Food, growth and exploitation of percids in Ohio's Upground Reservoirs. Trans. Am. Fish. Soc. Spec. Publ., $11: 270-$ 277.

Persson L., 1986. Effects of reduced interspecific competition on ressource utilization in perch (Perca fluviatilis). Ecology, 67 : 355-364

Persson L., 1987. Competition induced switch in young of the year perch (Perca fluviatilis) : an experimental test of ressource limitation. Env. Biol. Fish., 19 (3) : 235-239.

Persson L. et Greenberg L.A., 1990. Interspecific and intraspecific size class competition affecting ressource use and growth of perch (Perca fluviatilis). Oikos, 59 : 97-106

Pinkas L., Oliphant M.S. et Iverson I.L.K., 1971. Food habits of Albacore, Bluefin 
Tuna and Bonito in Californian waters. Calif. Fish. Game, 152 : 1-105.

Ponton D., 1986. Croissance et alimentation de deux poissons planctonophages du lac Lèman: le corégone (Coregonus schinzi palea, J.) et le gardon (Rutilus rutilus (L.)). Th. Doc. $3^{\text {e }}$ cycle. Ecologie des eaux, Univ. Cl. Bernard, Lyon I, $157 \mathrm{p}$.

Spanovskaya V.D. et Grygorash V.A., 1977. Development and food of age-0 Eurasian perch (Perca fluviatilis) in reservoirs near Moscow, URSS. J. Fish. Res. Board Can., 34 (10) : 1551-1558.

Swynnerton G.H. et Worthington E.B., 1940. Note on the food of fish in Haweswater (Westmorland). J. Anim. Ecol., 9 : 183-187.
Weatherley N.S., 1987. The diet and growth of 0-group dace, Leuciscus leuciscus (L.) and roach, Rutilus rutilus (L.) in a Lowland River. J. Fish. Biol., 30 : 237-247.

Werner E.E. et Hall D.J., 1974. Optimal foraging and size selection of prey by bluegill (Lepomis macrochirus). Ecology, $55: 1042-1052$

Zaret T.M., 1980. The effect of prey motion on planktivore choice. In: W.C. Kerfoot (ed.), Evolution and Ecology of Zooplankton Communities. The Univ. Press of New England, Hanover, New Hampshire, USA : 594-603.

Zaret T.M. et Kerfoot W.C., 1975. Fish predation on Bosmina longirostris: Bodysize selection versus visibility selection. Ecology, 56 : 232-237. 